\title{
Efficacy and safety of inetetamab in combination with chemotherapy as first-line treatment of HER2-positive metastatic breast cancer: a subgroup analysis in the HOPES study
}

\author{
Tao Wang ${ }^{1}$, Pin Zhang ${ }^{2}$, Lijun Di $^{3}$, Xiaojia Wang ${ }^{4}$, Junlan Yang ${ }^{5}$, Zhongsheng Tong ${ }^{6}$, Jian Liu ${ }^{7}$, Jifeng Feng ${ }^{8}$, \\ Donggeng Liu' ${ }^{9}$ Qitao $\mathbf{Y u}^{10}$, Yunpeng Liu $^{11}$, Hao Yu ${ }^{12}$, Zefei Jiang ${ }^{1}$ \\ ${ }^{1}$ Department of Oncology, the Fifth Medical Center of Chinese PLA General Hospital, Beijing, China; ${ }^{2}$ Department of Medical Oncology, Cancer \\ Hospital, Chinese Academy of Medical Sciences, Beijing, China; ${ }^{3}$ Department of Breast Oncology, Peking University Cancer Hospital \& Institute, \\ Beijing, China; ${ }^{4}$ Department of Medical Oncology, Zhejiang Cancer Hospital, Hangzhou, China; ${ }^{5}$ Department of Medical Oncology, the First \\ Medical Center of Chinese PLA General Hospital, Beijing, China; ${ }^{6}$ Department of Breast Oncology, Tianjin Medical University Cancer Institute \\ and Hospital, Tianjin, China; ${ }^{7}$ Department of Breast Medicine, Fujian Cancer Hospital, Fuzhou, China; ${ }^{8}$ Department of Medical Oncology, Jiangsu \\ Cancer Hospital, Nanjing, China; ${ }^{9}$ Department of Medical Oncology, Sun Yat-sen University Cancer Center, Guangzhou, China; ${ }^{10}$ Department of \\ Medical Oncology, Guangxi Medical University Affiliated Cancer Hospital, Nanning, China; ${ }^{11}$ Department of Medical Oncology, The First Hospital \\ of China Medical University, Shenyang, China; ${ }^{12}$ Department of Biostatistics, School of Public Health, Nanjing Medical University, Nanjing, China \\ Contributions: (I) Conception and design: Z Jiang; (II) Administrative support: Z Jiang; (III) Provision of study materials or patients: All authors (IV) \\ Collection and assembly of data: All authors; (V) Data analysis and interpretation: T Wang; (VI) Manuscript writing: All authors; (VII) Final approval \\ of manuscript: All authors. \\ Correspondence to: Zefei Jiang. Department of Oncology, the Fifth Medical Center of Chinese PLA General Hospital, Beijing 100070, China. \\ Email: jiangzefei@csco.org.cn.
}

Background: Our aim was to evaluate the efficacy and safety of inetetamab plus chemotherapy in the firstline treatment of HER2-positive metastatic breast cancer.

Methods: A HOPES study was conducted on patients with HER2-positive metastatic breast cancer. Eligible patients were randomly divided into test group and control group at a 2:1 ratio. Among them, patients in test group received inetetamab plus vinorelbine. Concurrently, patients in the control group received vinorelbine. During the trial, safety evaluation was conducted every 4 weeks and efficacy evaluation was conducted every 8 weeks. As assessed by the Response Criteria Evaluation in Solid Tumors (RECIST) 1.0 criteria, the primary endpoint was progression-free survival (PFS) and the secondary endpoints included objective response rate (ORR) and disease control rate (DCR). Safety was estimated according to the National Cancer Institute Common Toxicity Criteria (NCI-CTC) version 2.0. Efficacy and safety of the postoperative recurrent-metastases first-line subgroup in the HOPES registry study of inetetamab was analyzed and compared with previous clinical studies of trastuzumab in the first-line treatment of HER2positive metastatic breast cancer.

Results: In total, we included 315 patients. Among them, 114 patients in the postoperative recurrentmetastases first-line subgroup were assigned to the full analysis set (FAS) (test group, 72; and control group, 42). The test group significantly prolonged median PFS (mPFS) (11.1 vs. 3.3 months of the control group; $\mathrm{P}<0.0001)$. ORR and DCR were remarkably higher than the control group (ORR, 61.5\% vs. 29.7\% with an increase of $31.8 \%, \mathrm{P}=0.0224$; DCR, $93.8 \%$ vs. $59.4 \%$ with an increase of $34.4 \%, \mathrm{P}=0.0003$ ). Efficacy and safety of postoperative recurrent-metastases first-line subgroup in the HOPES study was comparable to previous clinical studies of trastuzumab as first-line treatment of HER2-positive metastatic breast cancer.

Conclusions: Inetetamab has shown efficacy and safety equivalent to trastuzumab for patients in the firstline treatment of postoperative recurrence-metastases HER2-positive breast cancer, which confirms its important status and potential as first-line treatment. Inetetamab provides more first-line targeted therapy options for patients with HER2-positive metastatic breast cancer. 
Keywords: Metastatic breast cancer; anti-HER2 targeted therapy; inetetamab; first-line treatment

Received: 28 December 2021; Accepted: 30 January 2022; Published: 30 April 2022.

doi: $10.21037 /$ tbcr-21-42

View this article at: https://dx.doi.org/10.21037/tbcr-21-42

\section{Introduction}

Breast cancer is one of the most common malignant tumors, in which HER2-positive cancer accounts for approximately $20 \%$ (1). Targeted therapy has been one of the most important roles in HER2-positive breast cancer treatment (2). Trastuzumab-based treatment remains a standard protocol for first-line treatment of HER2positive breast cancer. In 2017, real-world research report published online in the Oncologist by the American Society for Translational Oncology evaluated actual use in China and effectiveness of trastuzumab. The results show that unreasonable allocation of medical resources causes that usage rate of trastuzumab varies greatly in different regions regardless of in resource rich regions or not. Although trastuzumab treatment prolongs patients survival time, the actual use of trastuzumab in the early stage of breast cancer may affect the treatment after metastasis. The real-world results provide an opportunity to consider the optimization schemes of anti-HER2 treatment after metastasis, especially in areas where expensive targeted drugs are difficult to obtain (3). Currently, Chinese clinical practice and guidelines have a distance from the latest international treatment. There is still a huge unmet need for treatment. Selection limitations and high cost of antiHER2 mAb still need to be further improved (4). On this account, the Chinese government encourages and supports independently-developed innovative drugs to resolve practice requirement of breast cancer patients.

Inetetamab is a monoclonal antibody binding to domain IV of HER2 receptor. The Fab domain of inistumab is identical with trastuzumab, but whose amino acid sequence at positions 359 (D359, aspartic acid) and 361 (L361, leucine) is different from trastuzumab [E359 (glutamate) and M361 (methionine), respectively] in the constant region of the heavy chain of the $\mathrm{Fc}$ domain. In vitro data in the development stage indicated that inetetamab and trastuzumab presented same binding activity, comparable affinity with HER2 antigen and identical key quality attributes including inhibitory activity against in vitro cancer cell proliferation, protein folding, thermal stability, etc.

The HOPES study is a multi-center prospective randomized phase III trial used to evaluate efficacy and safety of inetetamab plus vinorelbine in treating HER2positive metastatic breast cancer patients with previous taxanes therapy and no anti-HER2 treatment (5). The patients in the test group received inetetamab plus vinorelbine, whereas the patients in the control group received vinorelbine. The test results show that inetetamab combined with vinorelbine significantly prolongs progression-free survival (PFS) and possesses excellent safety, compared with vinorelbine alone. Hence, inetetamab has been clinically approved for HER2-positive terminal breast cancer in 2020 and subsequently incorporated in the guidelines of breast cancer diagnosis and treatment of Chinese society of clinical oncology and the clinical practice guidelines of Breast Cancer of Chinese Anti-Cancer Association (6,7).

However, not all the subjects included in the HOPES study were first-line treatment, including patients with first- to fourth-line treatment. There was still no direct data to evaluate the status and potential of inetetamab in the first-line treatment of HER2-positive metastatic breast cancer. Therefore, the article aims to analyze efficacy and safety of a subgroup of first-line postoperative recurrencemetastases treatment in the HOPES study and compare previous clinical studies of trastuzumab combined with chemotherapy in the first-line treatment of HER2-positive metastatic breast cancer. And then efficacy and safety of inetetamab in the first-line treatment of HER2-positive metastatic breast cancer was investigated, providing more and better treatment options for Chinese breast cancer patients.

\section{Methods}

\section{Trial design}

This HOPES study was a randomized controlled, multicenter, prospective phase III clinical trial led by the Fifth Medical Center of Chinese PLA General Hospital along with 26 research centers across the country. Using centralized random grouping, the qualified patients were randomly assigned to the test group and the control group 
at a ratio of 2:1.

\section{Therapeutic methods}

Drugs used in this trial were inetetamab for injection (Cipterbin $^{\circledR}, 50 \mathrm{mg} /$ bottle, Sunshine Guojian Pharmaceutical, batch number: 20081201, 20090601) and vinorelbine bitartrate injection (Navelbine ${ }^{\circledR}, 10 \mathrm{mg} /$ bottle, Pierre Fabre Pharmaceuticals, batch number: 2AQ1083, 2AQ1086, 2AQ1087). Patients in test group received intravenous Cipterbin infused at a $4 \mathrm{mg} / \mathrm{kg}$ initial dose and then at a $2 \mathrm{mg} / \mathrm{kg}$ maintenance dose once per week, and combined with $25 \mathrm{mg} / \mathrm{m}^{2}$ of intravenous vinorelbine infused on day $1,8,15$ of every 28 days, until unacceptable progression of disease. If the patients in the test group developed chemotherapy toxicity intolerance, treatment continued intravenous Cipterbin infused at an $8 \mathrm{mg} / \mathrm{kg}$ initial dose and then at a $6 \mathrm{mg} / \mathrm{kg}$ maintenance dose once per 3 weeks, until disease progression. Patients in the control group received $25 \mathrm{mg} / \mathrm{m}^{2}$ of intravenous vinorelbine infused on day 1,8 , 15 of every 28 days until unacceptable disease progression or chemotherapy toxicity intolerance. After that, treatment continued intravenous Cipterbin infused at an $8 \mathrm{mg} / \mathrm{kg}$ initial dose and then at a $6 \mathrm{mg} / \mathrm{kg}$ maintenance dose once per 3 weeks, until disease progression.

\section{Patients}

Eligible patients were randomly assigned to test group and control group with a 2:1 ratio. Inclusion criteria were as follows: (I) aged 18-70 years; (II) histologically diagnosis of advanced breast cancer; (III) a centrally confirmed HER2positive status; (IV) clear measurable lesions as defined by the Response Evaluation Criteria in Solid Tumors (RECIST); (V) patients with central nervous system metastases during stable period were permitted to enrolled; (VI) previously untreated recurrence-metastases breast cancer.

\section{Trial endpoints}

The primary endpoint was PFS defined as the time from first medication to first recorded tumors progression or death from any cause.

The secondary endpoints included objective response rate (ORR) and disease control rate (DCR). ORR was defined as percentage of participants with complete response (CR) and partial response (PR). DCR was defined as percentage of participants with CR, $\mathrm{PR}$ and stable disease
(SD). Safety was estimated according to the National Cancer Institute Common Toxicity Criteria (NCI-CTC version 2.0), including adverse events (AE), serious AEs (SAE) and cardiotoxicity.

\section{Follow-up}

During the trial, the patients received regular vital signs, physical examination and related laboratory tests. Safety evaluation was conducted every 4 weeks and efficacy evaluation was conducted every 8 weeks. After the patients in the test group or the control group received Cipterbin alone, safety and efficacy were evaluated every 6 weeks.

\section{Statistical methods}

The sample size of the HOPES study is determined directly according to the regulation, not calculated based on statistical assumptions. According to the requirements of Drug Registration Regulation for clinical trial validation, the number of cases should be at least 100 pairs. This HOPES trial included 330 patients (test group, 220; and control group, 110), which complied with the regulatory requirements. In this study, we analyzed efficacy and safety of the subgroups in the HOPES study that meet the firstline treatment of postoperative recurrence-metastases.

Statistical analysis was carried out using SPSS 19.0. Kaplan-Meier method was used to estimate the survival curve. The log-rank test was used to compare median PFS (mPFS) between groups, among them $\mathrm{P}<0.05$ indicated that mPFS of test group was superior to that of control group. Cochran Mantel Haenszel (CMH) test was used to compare ORR and DCR between groups with the use of chi-square statistic by controlling the central effect. Clopper-Pearson method was used to calculate $95 \%$ CI of ORR and DCR. Normal approximation method was used to compute $95 \%$ CI of the ORR and DCR difference between groups. Descriptive statistical analysis was conducted for the data of safety (including AE) and laboratory. Fisher's exact test was used for subgroup comparisons. $\mathrm{P}$ values $<0.05$ were considered statistically significant.

\section{Ethical statement}

The study was conducted in accordance with the Declaration of Helsinki (as revised in 2013). The study was approved by National Medical Products Administration 


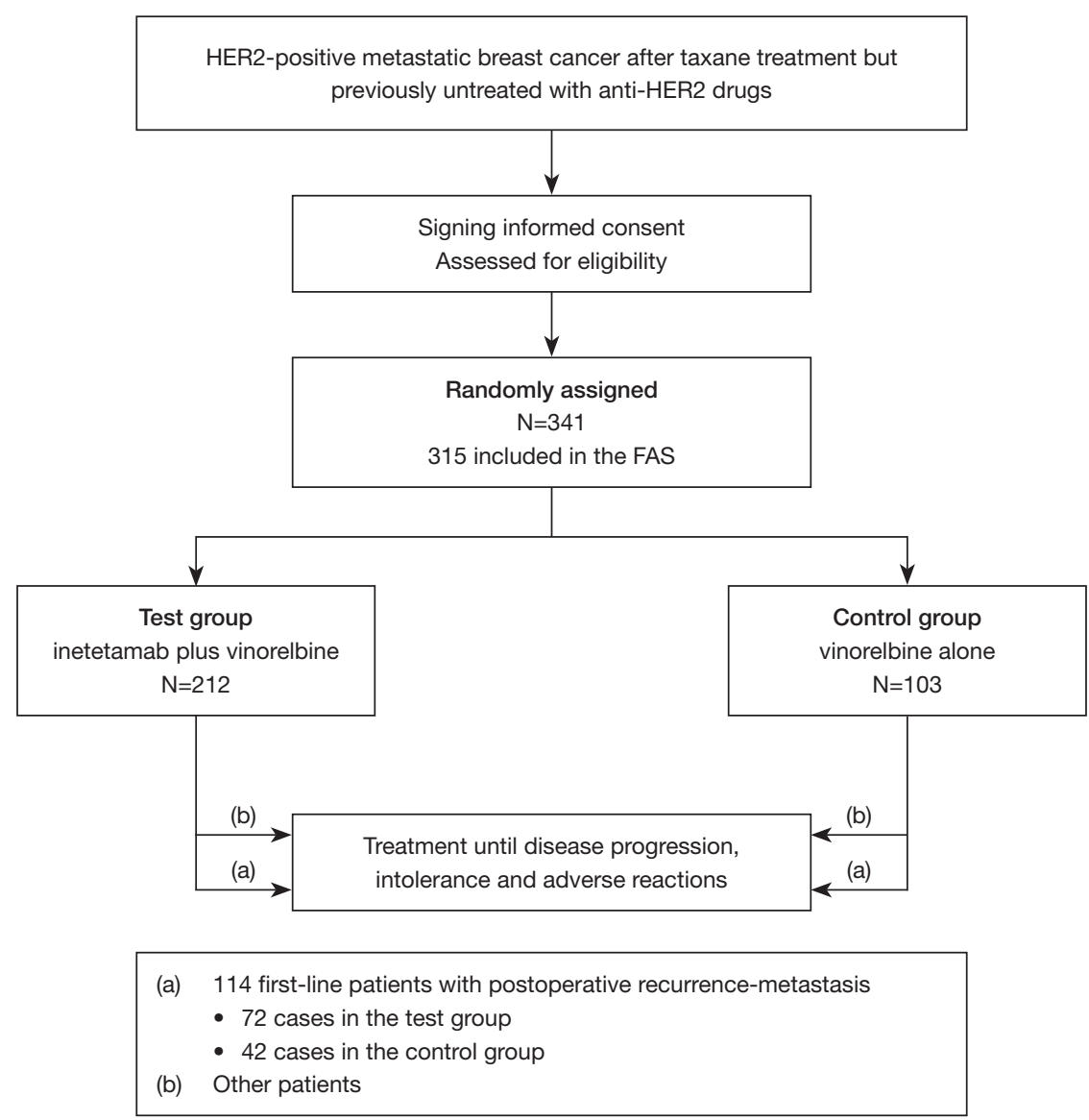

Figure 1 Trial profile. FAS, full analysis set.

(No. 2004L02352) and ethics board of the Fifth Medical Center of Chinese PLA General Hospital (No. 2009-03-10). Informed consent was obtained from all individual participants included in the study.

\section{Results}

We screened patients' data for meeting the first-line treatment in postoperative recurrence-metastases breast cancer in the subgroup of the HOPES registration study. 341 patients were randomly assigned and 315 patients were included in the full analysis set (FAS). Among them, 132 patients were eligible for the first-line treatment of postoperative recurrence-metastases: 114 patients included in the FAS (test group, 72; and control group, 42) and 126 patients included in the safety analysis set (SS) (test group, 81; and control group, 45) (Figure 1). Baseline characteristics of 114 subgroup patients with the first-line treatment was listed in Table 1. The baseline data of the test group and the control group were consistent, illustrating no statistical significance between groups $(\mathrm{P}>0.05)$.

As shown in Figure 2, the first-line PFS of patients who received inetetamab treatment $[11.1$ months; $95 \%$ CI: 8.25-not estimable (NE)] was significantly superior to vinorelbine control group (3.3 months; 95\% CI: 2.00-5.98; $\mathrm{P}<0.0001)$, the second-line and beyond PFS of patients who received inetetamab treatment (7.4 months; $95 \%$ CI: 5.72-9.72) was significantly superior to vinorelbine control group (2.8 months; 95\% CI: $1.84-4.37, \mathrm{P}<0.0001)$. Among them, the first-line PFS of patients with visceral metastases (11.1 months; 95\% CI: 7.49-16.00) exceeded obviously the control group (3.3 months; 95\% CI: 1.91-7.29; $\mathrm{P}<0.0001$ ). Especially the first-line PFS of patients with liver metastases (10.1 months; 95\% CI: 6.93-NE) exceeded obviously the control group (1.9 months; 95\% CI: 1.71-3.25; $\mathrm{P}<0.0001)$. Then the first-line PFS of patients with non-liver metastases (11.5 months; 95\% CI: 8.25-NE) was much longer than the control group (6.0 months; 95\% CI: 3.02-7.29; $\mathrm{P}=0.0048$ ) 
Table 1 First-line treatment subgroup baseline characteristics

\begin{tabular}{|c|c|c|c|}
\hline Characteristics & Inetetamab plus vinorelbine group $(n=72)$ & Vinorelbine control group $(n=42)$ & $\mathrm{P}$ \\
\hline Male & $2(2.8)$ & 0 & \\
\hline Female & $70(97.2)$ & $42(100.0)$ & \\
\hline Age (years), mean [range] & 50 [24-68] & 48.5 [30-69] & 0.8867 \\
\hline HER2 status, n (\%) & & & 0.3774 \\
\hline $\mathrm{IHC}(+++)$ & $53(73.6)$ & $36(85.7)$ & \\
\hline $\mathrm{IHC}(++), \mathrm{FISH}(+)$ & $5(6.9)$ & $2(4.8)$ & \\
\hline $\mathrm{IHC}(+), \mathrm{FISH}(+)$ & $3(4.2)$ & 0 & \\
\hline ER and/or PR positive & $26(36.1)$ & $18(42.9)$ & \\
\hline ER and PR negative & $46(63.9)$ & $24(57.1)$ & \\
\hline Lymph node metastases, n (\%) & & & 0.6229 \\
\hline 0 & $18(25.0)$ & $9(21.4)$ & \\
\hline $1-3$ & $16(22.2)$ & $12(28.6)$ & \\
\hline$\geq 4$ & $37(51.4)$ & $19(45.2)$ & \\
\hline Others & $1(1.4)$ & $1(2.4)$ & \\
\hline Missing & 0 & $1(2.4)$ & \\
\hline Others & $10(13.9)$ & $3(7.1)$ & \\
\hline Missing & $1(1.4)$ & 0 & \\
\hline Disease-free survival, $\mathrm{n}(\%)$ & & & 0.5273 \\
\hline$\leq 2$ years & $49(70.0)$ & $25(64.1)$ & \\
\hline$>2$ years & $21(30.0)$ & $14(35.9)$ & \\
\hline Treated previously with anthracyclines, n (\%) & & & 1.0000 \\
\hline Yes & $64(88.9)$ & $34(85.0)$ & \\
\hline No & $8(11.1)$ & $6(15.0)$ & \\
\hline
\end{tabular}

KPS, Karnofsky performance status; IHC, immunohistochemistry; FISH, fluorescence in situ hybridization; ER, estrogen receptor; PR, progesterone receptor. 


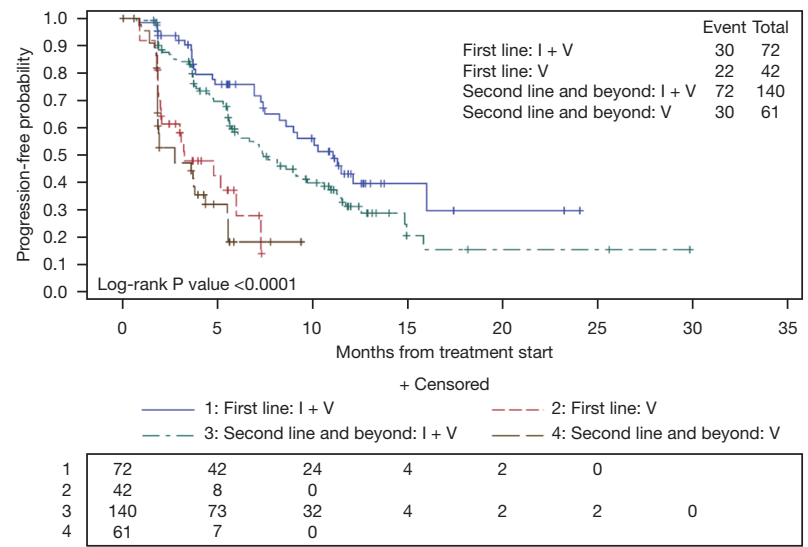

Figure 2 Kaplan-Meier curves of PFS in first-line, second-line and beyond subgroup. PFS, progression-free survival; $\mathrm{I}+\mathrm{V}$, inetetamab plus vinorelbine; $\mathrm{V}$, vinorelbine.

\section{(Figure 3).}

As listed in Table 2, ORR of the test group was remarkably superior to the control group $(61.5 \%$ vs. $29.7 \%$; $\mathrm{P}=0.0224$ ). Furthermore, $93.9 \%$ patients of the test group received disease control in the first-line treatment, also significantly higher than the control group $(\mathrm{P}=0.0003)$. ORR of the test group was remarkably superior to the control group (47.6\% vs. $17.8 \% ; \mathrm{P}=0.0008)$. Furthermore, $87.1 \%$ patients of the test group received disease control in the second-line and beyond treatment, significantly higher than the control group (55.6\%) $(\mathrm{P}=0.0002)$.

The adverse reactions in all enrolled patients were mostly hematologic and gastrointestinal toxicity (Table 3). The most common adverse reactions of inetetamab were decline of white blood cell count, whose incidence rate was $91.4 \%$ exceeding the control group $(\mathrm{P}=0.0309)$. The inetetamabrelated adverse reactions were mainly infusion-related fever and rigors, which were mostly grade 1 to 2 mild AEs. Safety was consistent with overall results of the HOPES study.

\section{Discussion}

The HOPES study was designed in 2009 for overcoming the dilemma that trastuzumab was unreachable due to expensive cost in China $(3,8)$. Subsequently trastuzumab and pertuzumab were approved for marketing in China and included into medical insurance, which solved the accessibility difficulties of Chinese people. However, inetetamab still can play an important role in special populations. These results in this study showed that mPFS of inetetamab in the first-line treatment was 11.1 months significantly longer than the control group. And patients with liver metastases might have better PFS benefit from inetetamab plus vinorelbine treatment. Inetetamab (clinical benefit rate $>90 \%$ ) had comparable anticancer efficacy with other historical data of trastuzumab, and was incapable to significantly increase the adverse reactions compared with the control group.

Antibody-dependent cell-mediated cytotoxicity (ADCC) is one of important mechanism of action of targeted monoclonal antibody (9). For instance, margetuximab is a Fc-modified anti-HER2 mAb, which has improved ADCC effect (10). The SOPHIA trial showed that margetuximab plus chemotherapy improved mPFS over trastuzumab (5.8 vs. 4.9 months) (11). Hence, the enhancement of ADCC can contribute to survival benefits. The Fab domain of inistumab is identical with trastuzumab, but whose amino acid sequence at positions 359 and 361 is different from trastuzumab in the constant region of the heavy chain of the Fc domain. The ADCC effect of Fc-modified inistumab is 1.11 times that of trastuzumab, which is one of the main reasons why inistumab shows an antitumor effect.

We analyzed efficacy of the subgroups in the HOPES study that meet the first-line treatment of postoperative recurrence-metastases. It turned out that inistumab plus vinorelbine significantly prolonged mPFS of firstline patients with postoperative recurrence-metastases, compared with vinorelbine alone. ORR and DCR of the test group also improved significantly, reaching statistical difference. In the registration study $\mathrm{H} 0648 \mathrm{~g}$ for the firstline treatment of HER2 metastatic breast cancer, the time to disease progression (TTP) of trastuzumab plus chemotherapy was 7.4 months and ORR was $50 \%$ (12). The M77001 study showed that TTP of trastuzumab plus docetaxel for treating the first-line patients was 11.7 months and effective rate was $61 \%$ (13). Furthermore, clinical trial of trastuzumab combined with vinorelbine in the firstline treatment of HER2-positive metastatic breast cancer demonstrated that TTP was 8.5-15.3 months and ORR was $51-59.3 \%$ (14). The HERNATA study showed that median TTP of trastuzumab plus vinorelbine in the firstline treatment was 15.3 months higher than other studies, which might be related to excluding patients with brain metastases (15). Efficacy of inetetamab was as close as the contemporaneous studies of trastuzumab in the first-line anti-HER2 treatment, showing the role of inetetamab in first-line treatment (16). This study displayed that the PFS of the first-line subgroup was superior to the test group in 

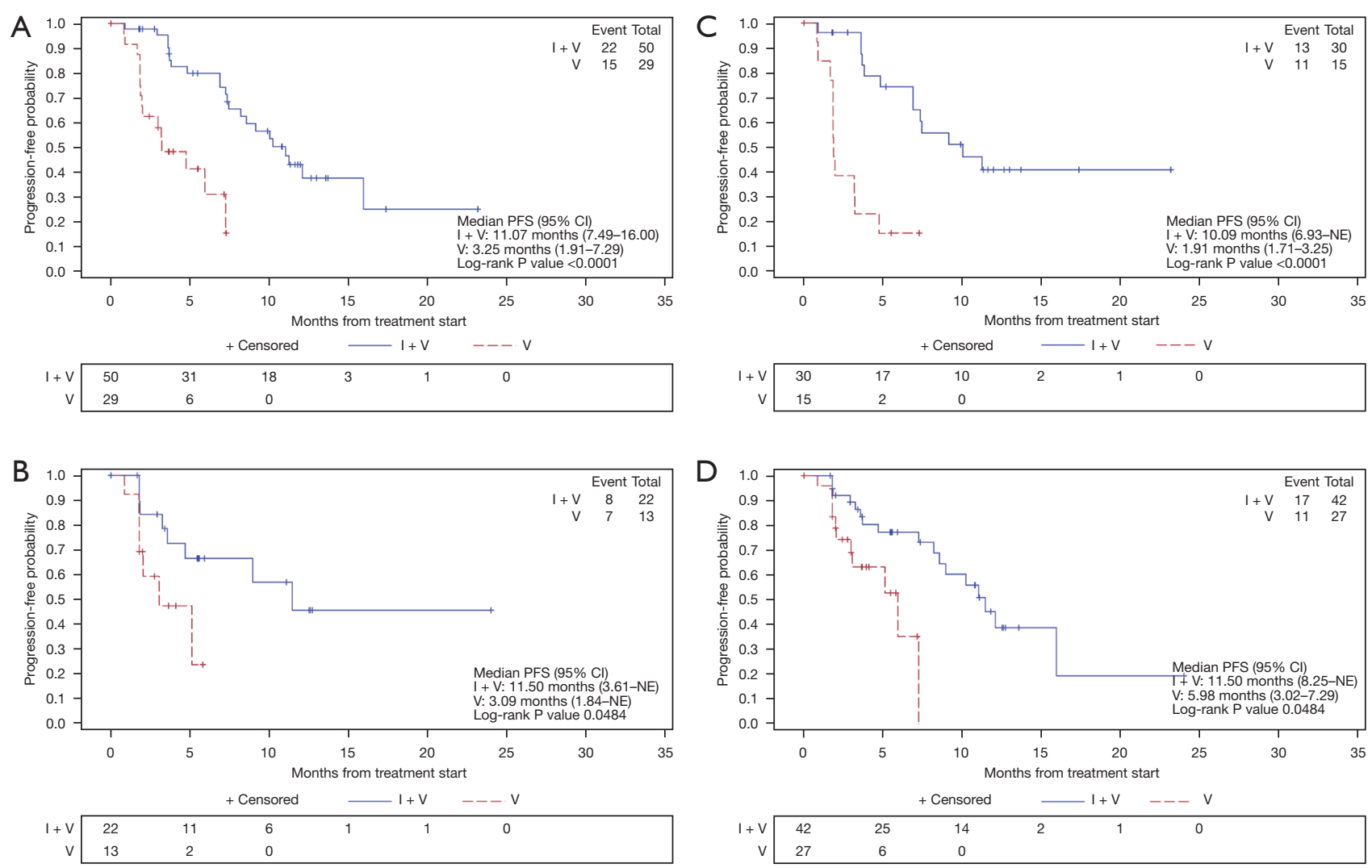

Figure 3 Kaplan-Meier curves of PFS in first-line subgroup of (A) visceral metastases, (B) non-visceral metastases, (C) liver metastases, (D) non-liver metastases. PFS, progression-free survival; $\mathrm{I}+\mathrm{V}$, inetetamab plus vinorelbine; $\mathrm{V}$, vinorelbine.

Table 2 Summary of efficacy between first-line treatment arms

\begin{tabular}{|c|c|c|c|}
\hline Outcomes & Inetetamab plus vinorelbine group & Vinorelbine control group & $\mathrm{P}$ \\
\hline PR, n (\%) & $35(53.8)$ & $10(27.0)$ & - \\
\hline $\mathrm{SD}, \mathrm{n}(\%)$ & $21(32.3)$ & $11(29.7)$ & - \\
\hline PD, n (\%) & $4(6.2)$ & $15(40.5)$ & - \\
\hline DCR (\%) (95\% Cl) & $93.85(84.99,98.30)$ & $59.46(42.10,75.25)$ & 0.0003 \\
\hline
\end{tabular}

$\mathrm{CR}$, complete response; PR, partial response; SD, stable disease; PD, progressive disease; ORR, objective response rate; DCR, disease control rate.

the HOPES study (11.1 vs. 9.1 months), which indicated that the different lines of chemotherapy treatment could affect the efficacy of anti-HER2 treatment to some extent. For the above-mentioned reasons, anti-HER2 treatment should be started as early as possible. Efficacy analyses for PFS in subgroups revealed that the patients with liver metastases might have better PFS benefit from inetetamab, which further hinted the dominant treatment population of inetetamab.

Safety of the first-line treatment was consistent with the HOPES study that vinorelbine plus inetetamab in the firstline treatment of patients with postoperative recurrence- 
Table 3 Incidence of adverse reactions (\% of patients)

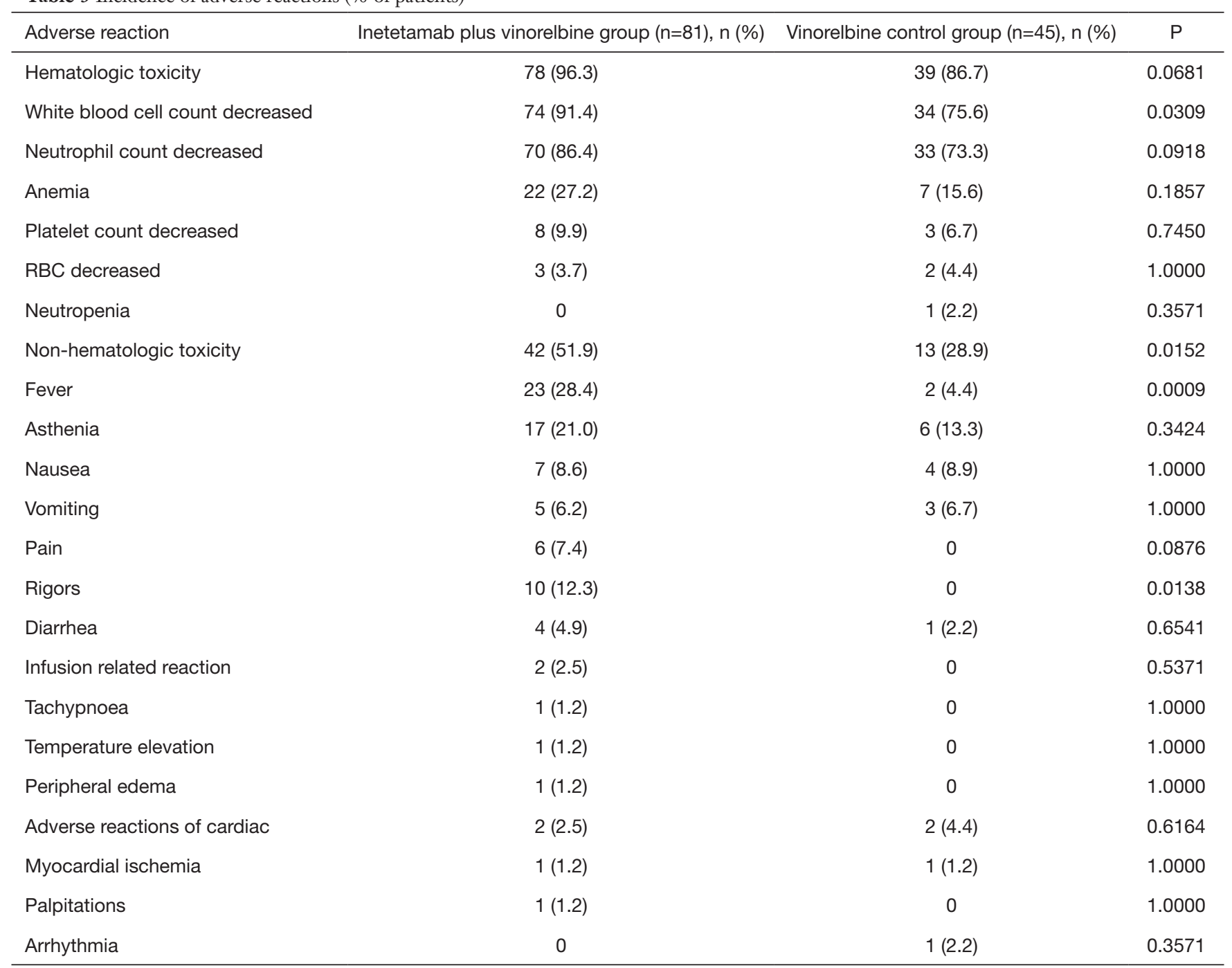

$\mathrm{RBC}$, red blood cell.

metastases did not significantly increase serious toxicity. Compared with the previous safety study of trastuzumab in the first-line treatment of HER2-positive metastatic breast cancer, it was not found that the first-line subgroup of the HOPES study for postoperative recurrence-metastases could cause new signals and forms of AEs. Meanwhile there were no significant change in grades and incidences of AEs, showing that inetetamab and trastuzumab are equivalently safe.

There remain several following defects in this study. Firstly, the control group was treated with chemotherapy alone. Although inetetamab showed good anticancer efficacy, whether it could benefit more than trastuzumab needs further research. Secondly, with regard to a subgroup analysis, a relatively small number of patients involved in this subgroup that it was impossible to obtain more rigorous conclusions. We will explore the real effect of inetetamab in first-line treatment through following real-world research.

\section{Conclusions}

Efficacy and safety of a subgroup of first-line postoperative recurrence-metastases treatment in the HOPES study are comparable to previous clinical studies of trastuzumab in the first-line treatment of HER2-positive advanced breast cancer. Inetetamab has demonstrated outstanding efficacy and safety, providing more options for the first-line 
treatment of HER2-positive patients.

\section{Acknowledgments}

The authors are grateful to the patients, clinicians, pathologists and statisticians who participated in this study. Funding: This study was supported by the National HighTech R\&D Program (863 Program) (No. 2006AA02A246).

\section{Footnote}

Data Sharing Statement: Available at https://tbcr.amegroups. com/article/view/10.21037/tbcr-21-42/dss

Conflicts of Interest: All authors have completed the ICMJE uniform disclosure form (available at https://tbcr. amegroups.com/article/view/10.21037/tbcr-21-42/coif). ZJ serves as the Editor-in-Chief of Translational Breast Cancer Research from November 2019 to October 2024. XW serves as an unpaid Editorial Board Member of Translational Breast Cancer Research from December 2020 to November 2022. The other authors have no conflicts of interest to declare.

Ethical Statement: The authors are accountable for all aspects of the work in ensuring that questions related to the accuracy or integrity of any part of the work are appropriately investigated and resolved. The study was conducted in accordance with the Declaration of Helsinki (as revised in 2013). The study was approved by National Medical Products Administration (No. 2004L02352) and ethics board of the Fifth Medical Center of Chinese PLA General Hospital (No. 2009-03-10). Informed consent was obtained from all individual participants included in the study.

Open Access Statement: This is an Open Access article distributed in accordance with the Creative Commons Attribution-NonCommercial-NoDerivs 4.0 International License (CC BY-NC-ND 4.0), which permits the noncommercial replication and distribution of the article with the strict proviso that no changes or edits are made and the original work is properly cited (including links to both the formal publication through the relevant DOI and the license). See: https://creativecommons.org/licenses/by-nc-nd/4.0/.

\section{References}

1. Sung H, Ferlay J, Siegel RL, et al. Global Cancer Statistics 2020: GLOBOCAN Estimates of Incidence and Mortality
Worldwide for 36 Cancers in 185 Countries. CA Cancer J Clin 2021;71:209-49.

2. Waks AG, Winer EP. Breast Cancer Treatment: A Review. JAMA 2019;321:288-300.

3. Li J, Wang S, Wang Y, et al. Disparities of Trastuzumab Use in Resource-Limited or Resource-Abundant Regions and Its Survival Benefit on HER2 Positive Breast Cancer: A Real-World Study from China. Oncologist 2017;22:1333-8.

4. Chinese Society of Clinical Oncology; Chinese AntiCancer Association; Committee of Breast Cancer Society. Expert consensus on diagnosis and management of human epidermal growth factor receptor 2 positive breast cancer (version 2021). National Medical Journal of China 2021;101:1226-31.

5. Bian L, Xu BH, Di LJ, et al. Phase III randomized controlled, multicenter, prospective study of recombinant anti-HER2 humanized monoclonal antibody (Cipterbin) combined with vinorelbine in patients with HER2 positive metastatic breast cancer: the HOPES Study. National Medical Journal of China 2020;100:2351-7.

6. Guidelines Working Committee of Chinese Society of Clinical Oncology. Chinese Society of Clinical Oncology Breast Cancer Guideline, 2021 ver. Beijing: People's Medical Publishing House, 2021.

7. Chinese Anti-Cancer Association; Committee of Breast Cancer Society. Clinical Practice Guidelines in Breast Cancer by Chinese Anti-Cancer Association (2021 version). China Oncology 2021;31:954-1040.

8. Reeder-Hayes K, Peacock Hinton S, Meng K, et al. Disparities in Use of Human Epidermal Growth Hormone Receptor 2-Targeted Therapy for Early-Stage Breast Cancer. J Clin Oncol 2016;34:2003-9.

9. Collins DM, Madden SF, Gaynor N, et al. Effects of HER Family-targeting Tyrosine Kinase Inhibitors on Antibodydependent Cell-mediated Cytotoxicity in HER2-expressing Breast Cancer. Clin Cancer Res 2021;27:807-18.

10. Bang YJ, Giaccone G, Im SA, et al. First-in-human phase 1 study of margetuximab (MGAH22), an Fc-modified chimeric monoclonal antibody, in patients with HER2positive advanced solid tumors. Ann Oncol 2017;28:855-61.

11. Rugo HS, Im SA, Cardoso F, et al. Efficacy of Margetuximab vs Trastuzumab in Patients With Pretreated ERBB2-Positive Advanced Breast Cancer: A Phase 3 Randomized Clinical Trial. JAMA Oncol 2021;7:573-84.

12. Slamon DJ, Leyland-Jones B, Shak S, et al. Use of chemotherapy plus a monoclonal antibody against HER2 for metastatic breast cancer that overexpresses HER2. N 
Engl J Med 2001;344:783-92.

13. Marty M, Cognetti F, Maraninchi D, et al. Randomized phase II trial of the efficacy and safety of trastuzumab combined with docetaxel in patients with human epidermal growth factor receptor 2-positive metastatic breast cancer administered as first-line treatment: the M77001 study group. J Clin Oncol 2005;23:4265-74.

14. Farhat F, Kattan JG, Ghosn M. Oral vinorelbine in combination with trastuzumab as a first-line therapy of metastatic or locally advanced HER2-positive breast

doi: $10.21037 /$ tbcr-21-42

Cite this article as: Wang T, Zhang $\mathrm{P}$, Di L, Wang X, Yang J, Tong Z, Liu J, Feng J, Liu D, Yu Q, Liu Y, Yu H, Jiang Z. Efficacy and safety of inetetamab in combination with chemotherapy as first-line treatment of HER2-positive metastatic breast cancer: a subgroup analysis in the HOPES study. Transl Breast Cancer Res 2022;3:15. cancer. Cancer Chemother Pharmacol 2016;77:1069-77.

15. Andersson M, Lidbrink E, Bjerre K, et al. Phase III randomized study comparing docetaxel plus trastuzumab with vinorelbine plus trastuzumab as first-line therapy of metastatic or locally advanced human epidermal growth factor receptor 2-positive breast cancer: the HERNATA study. J Clin Oncol 2011;29:264-71.

16. Oh DY, Bang YJ. HER2-targeted therapies - a role beyond breast cancer. Nat Rev Clin Oncol 2020;17:33-48. 